\title{
Cholinergic Enhancement Increases the Effects of Voluntary Attention but Does Not Affect Involuntary Attention
}

\author{
Ariel Rokem*', ${ }^{*}$ Ayelet N Landau' ${ }^{2}$, Dave Garg', William Prinzmetal ${ }^{2}$ and Michael A Silver ${ }^{1,3}$ \\ 'Helen Wills Neuroscience Institute, University of California, Berkeley, CA, USA; ${ }^{2}$ Department of Psychology, University of California, Berkeley, \\ CA, USA; ${ }^{3}$ School of Optometry, University of California, Berkeley, CA, USA
}

\begin{abstract}
Voluntary visual spatial attention can be allocated in a goal-oriented manner to locations containing behaviorally relevant information. In contrast, involuntary attention is automatically captured by salient events. Allocation of attention is known to be modulated by release of the neurotransmitter acetylcholine (ACh) in cerebral cortex. We used an anti-predictive spatial cueing task to assess the effects of pharmacological enhancement of cholinergic transmission on behavioral measures of voluntary and involuntary attention in healthy human participants. Each trial began with the presentation of a cue in a peripheral location. In $80 \%$ of the trials, a target then appeared in a location opposite the cue. In the remaining $20 \%$ of trials, the target appeared in the cue location. For trials with short stimulus onset asynchrony (SOA) between cue and target, involuntary capture of attention resulted in shorter reaction times (RTs) to targets presented at the cue location. For long SOA trials, allocation of voluntary attention resulted in the opposite pattern: RTs were shorter when the target appeared in the expected (opposite) location. Each subject participated in two sessions: one in which the cholinesterase inhibitor donepezil was administered to increase synaptic ACh levels and one in which placebo was administered. Donepezil selectively improved performance (reduced RT) for long SOA trials in which targets appeared in the expected location. Thus, cholinergic enhancement augments the benefits of voluntary attention but does not affect involuntary attention, suggesting that they rely on different neurochemical mechanisms.

Neuropsychopharmacology (2010) 35, 2538-2544; doi:I0.1038/npp.2010.1 I8; published online I September 20I0
\end{abstract}

Keywords: acetylcholine; donepezil; involuntary attention; spatial attention; visual attention; voluntary attention

\section{INTRODUCTION}

Selection of incoming sensory information is required for effective processing, and visual spatial attention is one mechanism by which certain regions of space are selected for processing over others (Posner et al, 1982; Prinzmetal and Landau, 2010). Two distinct types of visual spatial attention have been identified. On the one hand, attention can be voluntarily allocated to a location that is relevant for performing a task (Posner et al, 1982). On the other hand, attention can be captured in an involuntary manner by a salient event at a spatial location, even when that location is not task relevant (Yantis and Jonides, 1990). These two forms of attention (voluntary and involuntary, or endogenous and exogenous) have different consequences for the processing of visual stimuli (Prinzmetal et al, 2005; Prinzmetal et al, 2008) and are associated with different neural mechanisms (Kincade et al, 2005; Landau et al, 2007; Esterman et al, 2008). In addition, voluntary and involun-

*Correspondence: Dr A Rokem, Helen Wills Neuroscience Institute, University of California, Berkeley, 360 Minor Hall \#2020, Berkeley, CA 94720-2020, USA, Tel: + I 510642 3134, Fax: + I 5106435 I09, E-mail: arokem@berkeley.edu

Received 20 April 2010; revised 24 June 2010; accepted 8 July 2010 tary attention differ in their time course. Involuntary attention is fast to develop but is transient, dissipating quickly (Posner et al, 1982). In contrast, voluntary attention takes more time to develop (Posner et al, 1982; Prinzmetal and Landau, 2010), but can be sustained for many seconds (Silver et al, 2007).

We examined the role of the neurotransmitter acetylcholine (ACh) in modulating voluntary and involuntary attention in healthy human subjects. ACh has been found to facilitate cognitive processes such as attention and learning (Sarter et al, 2005). Cholinergic neurons in the basal forebrain project widely to cerebral cortex, where they release ACh when animals are performing attentionally demanding tasks (Arnold et al, 2002). Conversely, performance in such tasks is impaired when the basal forebrain nuclei are lesioned (Muir et al, 1994). Cholinesterase inhibitors such as donepezil and physostigmine increase synaptic levels of ACh by inhibiting the enzymatic breakdown of ACh in the synaptic cleft, and physostigmine has been reported to improve performance on a voluntary visual attention task (Bentley et al, 2004).

We used an anti-cueing task (Posner et al, 1982; Warner et al, 1990; Sereno and Holzman, 1996) (Figure 1) to separately measure the effects of voluntary and involuntary 


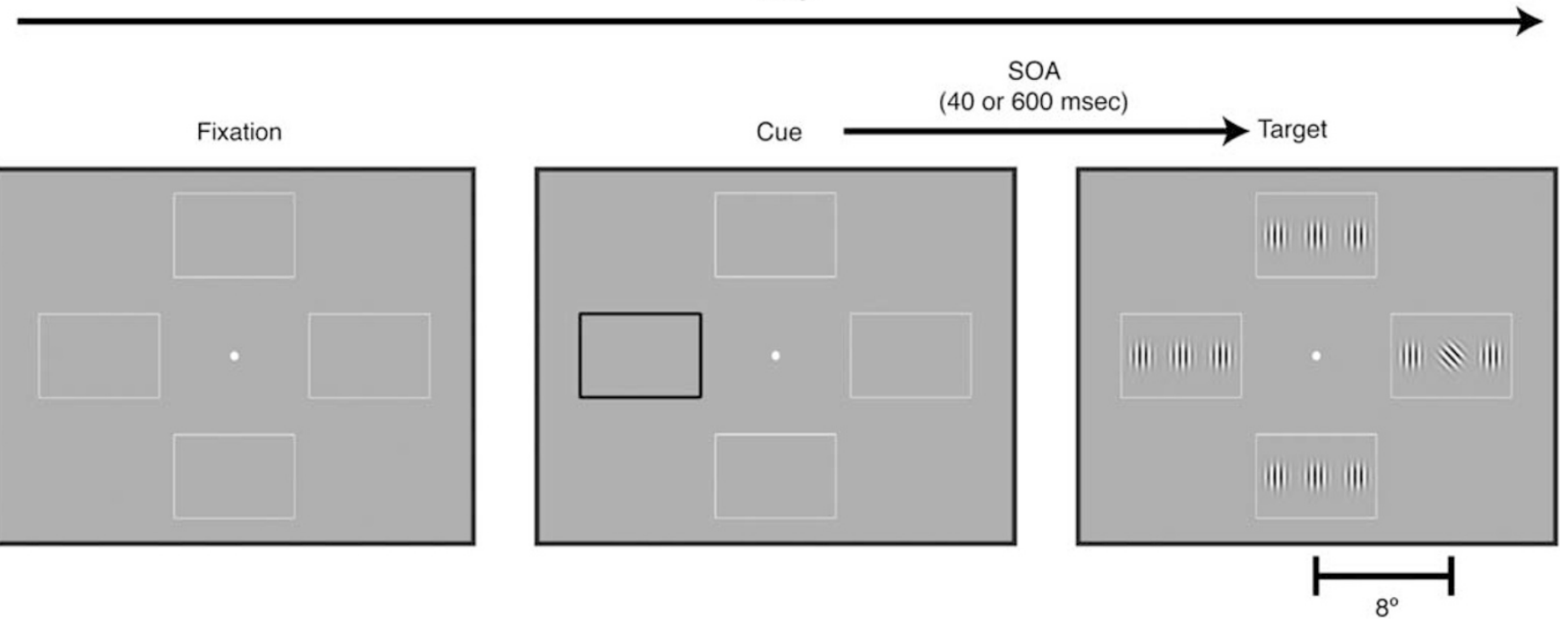

Figure I Anti-cueing task. At the beginning of each trial, one of the four peripheral rectangular frames became black and thicker. This cue indicated that the target would be most likely (80\%) to appear in the opposite location after a stimulus onset asynchrony (SOA) of either 40 or 600 ms. In the remaining $20 \%$ of trials, the target appeared at the cue location. The target was a Gabor patch oriented $\pm 45^{\circ}$ relative to vertical. Subjects indicated target orientation as quickly and accurately as they could by pressing one of two buttons.

attention on behavioral performance. A double-blind placebo-controlled crossover design was used to assess the effects of donepezil on these two types of attention. The use of an anti-cueing task in this study generated separate measurements of the effects of voluntary and involuntary attention on behavioral performance, and the withinsubject design allowed assessment of the effects of cholinergic enhancement on these two types of attention. We found that cholinergic enhancement increased the benefits in performance due to voluntary attention but had no effect on involuntary attention.

\section{MATERIALS AND METHODS}

\section{Subjects}

The experimental procedures were approved by the Committee for the Protection of Human Subjects at the University of California, Berkeley. There were 20 participants (10 females, mean age: $23 \pm 3$ years), all of whom had normal or corrected-to-normal vision. Tobacco smokers were excluded. Subjects were paid for their participation.

\section{Procedure}

Each subject participated in three sessions. In the first session, a health screen was conducted and informed consent was obtained. Then, subjects were acquainted with the behavioral task and practiced it for 500 trials. Before each of the subsequent sessions, subjects were administered a pill containing either $5 \mathrm{mg}$ donepezil or placebo. A crossover design was used in which each subject received placebo on one session and donepezil on the other. Drug administration was double-blind, and the order of drug and placebo administration was counterbalanced between subjects. For each of the drug and placebo sessions, subjects performed 1000 trials of the task (approximately $1 \mathrm{~h}$ of testing). Testing started $3 \mathrm{~h}$ after the pill was administered, corresponding to the time of peak plasma concentration of donepezil after oral ingestion (Rogers and Friedhoff, 1998). At least 2 weeks passed between the second and third sessions, allowing the drug, if present, to be eliminated (the half-life of donepezil is approximately 80 hours (Rogers and Friedhoff, 1998)).

While performing the task, participants were seated in a dark room, with their eyes $50 \mathrm{~cm}$ from the display and their chin placed in a chin rest. They were instructed to fixate on a central point, and eye movements were monitored with an infrared camera. Subjects received auditory feedback at the end of a trial if they failed to maintain fixation, and trials containing eye movements away from the central fixation point were excluded from further analysis. Donepezil has previously been found to have no effect on fixation stability at the 5-mg dose used in the present study (Silver et al, 2008). The proportion of trials in which eye movements occurred was generally low ( $0.5 \%$ of all trials) and did not differ between drug and placebo sessions $\left(F_{1,18}=0.1\right.$, $p=0.8)$.

\section{Task}

An anti-cueing task was used to dissociate voluntary and involuntary attention (Posner et al, 1982; Warner et al, 1990; Sereno and Holzman, 1996) (Figure 1). Each trial began with a 200-ms cue: one of the peripheral rectangular frames became black and slightly thicker (from 0.1 to $0.24^{\circ}$ of visual angle). The appearance of this cue in one location predicted the appearance of a target grating in the opposite location for $80 \%$ of the trials and in the same location as the cue for the remaining $20 \%$ of trials. The target display contained 12 Gabor patches (100\% contrast, spatial frequency of two cycles per degree; space constant of 
$0.8^{\circ}$ ), three within each frame. The target (always the central of the three Gabor patches) was tilted $\pm 45^{\circ}$ away from vertical, and all other patches were vertically oriented. Subjects were instructed to report the direction of tilt of the target by pressing a button as accurately and as quickly as they could. Auditory feedback on performance was provided at the end of each trial. In different blocks (250 trials per block), the stimulus onset asynchrony (SOA) between the appearance of the cue and the appearance of the target was either 40 or $600 \mathrm{~ms}$. The 40 - and $600-\mathrm{ms}$ SOA blocks were interleaved, and the order of conditions was counter-balanced between subjects, such that all combinations of order of SOA and order of drug administration were equally represented. The target display appeared for $133 \mathrm{~ms}$ in 40-ms SOA blocks and for $333 \mathrm{~ms}$ in 600-ms SOA blocks. This difference in target duration was the consequence of an inadvertent programming error. However, it is not likely to have affected any conclusions regarding the effects of cholinergic enhancement. Robust effects of the cue were observed in the expected direction for both SOAs, and the effects of donepezil were tested within each combination of SOA and target location. Visual stimuli were presented on a CRT monitor, using the Psychophysics Toolbox (Brainard, 1997; Pelli, 1997).

\section{Analysis}

Trials with RTs faster than $100 \mathrm{~ms}$ or slower than $1000 \mathrm{~ms}$ were excluded from analysis, as were trials with RTs more than three SD values away from the mean for that condition. On average, $2.5 \%$ of the trials in each block were excluded using these criteria. Trials with incorrect responses were also excluded. Mean RTs were analyzed in a mixed-model ANOVA. Cue (target in the cue or opposite location), drug (placebo or donepezil), and SOA (40 or $600 \mathrm{~ms}$ ) were entered as within-subject factors, and order (placebo first or donepezil first) was entered as a between-subject factor.

Additional planned comparisons were conducted to address specific hypotheses. Analysis of variance partitions the variance for each combination of within-subject factors (combination of drug condition, SOA, and target location) into variance due to the order of drug administration (the between-subject factor) and variance due to noise. Only the variance due to noise is relevant for assessing the statistical significance of the within-subject factors, and this is the error term used in our planned comparisons. These comparisons are made by calculating the contrast in question (eg, long SOA, cue location in the placebo session $v s$ long SOA, cue location in the donepezil session) and normalizing the resulting quantity by a measure of error based on the highest-order interaction in the ANOVA (Keppel and Wickens, 2004). To test the null hypothesis (in this example, no effect of the drug), this normalized contrast value is compared with the Student's $t$-distribution, and the degrees of freedom are associated with the error of the highest-order interaction in the ANOVA.

To control for type I error in the planned comparisons, we used a family-wise Bonferroni correction for multiple comparisons (Keppel and Wickens, 2004). This correction was separately applied to each combination of factors in the ANOVA for which a planned comparison was conducted (four different cueing effects, four different drug effects, and two different interactions of drug and cueing). All statistical tests were based on non-directional (two-tailed) null hypotheses.

\section{RESULTS}

An anti-cueing task (Posner et al, 1982; Warner et al, 1990; Sereno and Holzman, 1996) was used to measure the effects of voluntary and involuntary attention. Each trial began with a cue in one of four locations, predicting the subsequent appearance of the target in the opposite location for $80 \%$ of the trials (Figure 1). In the remaining $20 \%$ of trials, the target appeared in the same location as the cue. For all trials, involuntary attention is initially drawn to the appearance of the salient cue. With increasing time following cue presentation, voluntary attention can be allocated to the opposite location (where the target was presented for $80 \%$ of the trials). To separately examine the effects of involuntary and voluntary attention, SOA between the cue and target was varied between blocks.

We first describe the effects of spatial cueing after placebo administration. For half of the blocks, the SOA was $40 \mathrm{~ms}$, corresponding to an interval for which involuntary attention is still present in most subjects, but voluntary attention has not yet been allocated (Posner et al, 1982). In these blocks, reaction times (RTs) to target presentation were faster in the cue location (518 ms, SD: $75 \mathrm{~ms}$ ) compared with the opposite location (538 ms, SD: $82 \mathrm{~ms}$ ) (Figure 2). Notice that between-subject variability in reaction times is rather large. To isolate the effects of attention independent of overall RT differences between subjects, we calculated the

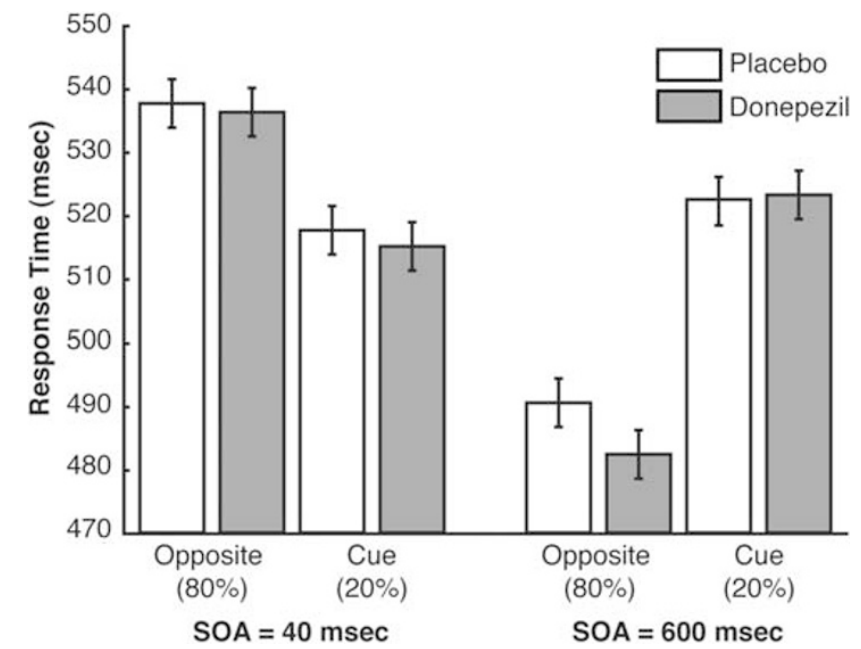

Figure 2 Effects of target location, stimulus onset asynchrony (SOA), and drug condition on reaction times (RTs). RTs in placebo (white) and donepezil (gray) sessions. For trials in which the SOA was $40 \mathrm{~ms}$ (left), RTs were significantly faster for the $20 \%$ of trials in which the target appeared in the same location as the cue, indicating capture of involuntary attention. For trials in which the SOA was 600 ms (right), RTs were significantly faster for the $80 \%$ of trials in which the target appeared in the opposite location, indicating allocation of voluntary attention. Donepezil reduced RTs in only one of the four conditions: $600 \mathrm{~ms}$ SOA trials in which the target appeared in the location opposite to the cue. Error bars are within-subject errors, calculated from the error term in the highest-order interaction in the analysis of variance (Loftus and Masson, 1994). 


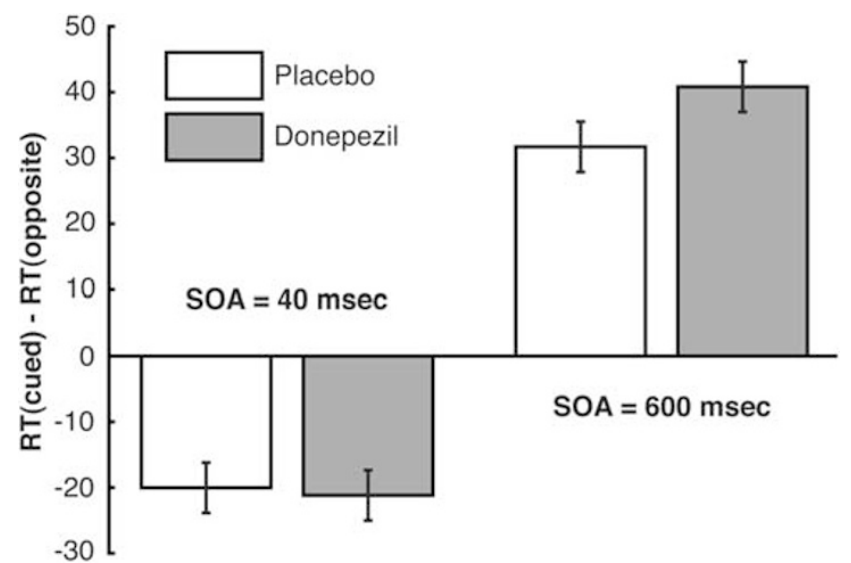

Figure 3 Cholinergic enhancement increases the cueing effect for voluntary but not involuntary attention. The cueing effect is defined as the difference between the mean reaction time (RT) for trials in which the target appeared at the cue location and the mean RT for trials in which the target appeared at the opposite location. Cueing effects were computed for each subject in each condition, and the mean cueing effects are presented for placebo (white) and donepezil (gray). For 40-ms stimulus onset asynchrony (SOA) (left), the cueing effect was negative, indicating capture of involuntary attention at the cue location. For 600-ms SOA (right), the cueing effect was positive, indicating that voluntary attention was allocated to the location opposite the cue. Cholinergic enhancement increased the magnitude of the cueing effect only for the 600-ms SOA trials. Error bars are as in Figure 2.

magnitude of the within-subject cueing effect, defined here as the difference between the mean RT when the target was in the cue location and the mean RT when the target was in the opposite location. The mean cueing effect for short SOA trials was $-20 \mathrm{~ms}$ and was significantly less than zero (planned comparison, $\mathrm{t}_{18}=8.2, p<0.05$, corrected for multiple comparisons; Figure 3). This measure was negative in 17 of 20 subjects, suggesting that involuntary attention was successfully captured in the $40-\mathrm{ms}$ SOA condition in most of the participants.

In the remaining blocks, the SOA was $600 \mathrm{~ms}$. This allowed sufficient time for involuntary attention in the cue location to dissipate and for allocation of voluntary attention to the opposite location, where the target was likely to appear ( $80 \%$ probability). In these blocks, the mean RT was faster when the target appeared in the opposite location (491 ms, SD: $62 \mathrm{~ms}$ ) compared with the cue location (522 ms, SD: $59 \mathrm{~ms}$ ) (Figure 2). The cueing effect for these long SOA trials was positive for all 20 subjects, as was the average cueing effect (32 ms, planned comparison, $\mathrm{t}_{18}=12.7, p<0.01$, corrected for multiple comparisons; Figure 3). Moreover, ANOVA indicated that the interaction of SOA and cue was significant $\left(\mathrm{F}_{1,18}=75.4, p<0.01\right)$, demonstrating that short and long SOAs produced different patterns of RTs. Indeed, the difference between the cueing effect in the long and short SOA conditions was positive for all 20 subjects (mean $=52 \mathrm{~ms}$ ). These results (in the placebo condition) replicate previous findings obtained using the anti-cueing procedure (Posner et al, 1982; Warner et al, 1990; Sereno and Holzman, 1996).

To test the effects of ACh on voluntary and involuntary attention, the cholinesterase inhibitor donepezil was administered. Each subject received $5 \mathrm{mg}$ donepezil before one session and placebo before the other session. There was no main effect of drug administration on overall RT $\left(\right.$ placebo $=517 \mathrm{~ms}$; donepezil $=514 \mathrm{~ms} ; \mathrm{F}_{1,18}=0.3, p=0.6$ ). Similar to the placebo condition, the cueing effect was significant under donepezil for both the short (planned comparison, $\mathrm{t}_{18}=8.7, p<0.01$ ) and long SOA (planned comparison, $\mathrm{t}_{18}=16.7, p<0.01$, both corrected for multiple comparisons).

To test the effects of cholinergic enhancement, planned comparisons were conducted for all SOA/target location combinations, and donepezil had a significant effect in only one of these four conditions: SOA of $600 \mathrm{~ms}$ and target in the opposite location as the cue (placebo $=491 \mathrm{~ms}$; donepezil $=483 \mathrm{~ms} ; \mathrm{t}_{18}=3.3, p<0.05$, corrected for multiple comparisons). No effect of the drug was found in planned comparisons for the other three conditions. Furthermore, ANOVA showed that the three-way interaction of drug administration, target location, and SOA was also significant $\left(\mathrm{F}_{1,18}=4.4, p<0.05\right)$. Finally, in the long SOA condition, the cueing effect was larger for donepezil than for placebo (planned comparison, $\mathrm{t}_{18}=2.6, p<0.05$, corrected for multiple comparisons), providing further evidence that cholinergic enhancement augmented voluntary but not involuntary attention. Taken together, the RT and cueing effect results demonstrate that cholinergic enhancement increased the effects of voluntary attention on performance. In particular, there was a selective advantage when voluntary attention enhanced processing of the stimulus - namely, when there was sufficient time for voluntary attention to be deployed and when the target was presented in the opposite (attended) location.

The lack of a main effect of the drug on overall RT or on any SOA/target location combinations other than long SOA/ opposite location rules out the possibility that the drug had a nonspecific overall effect on performance. Moreover, the high degree of similarity in task demands and stimulus characteristics across SOAs and target locations suggests that the drug effects measured at long SOAs reflect a specific enhancement of voluntary allocation of attention (rather than interactions between drug and stimulus properties).

A significant interaction of session order and drug $\left(F_{1,18}=33.3, p<0.01\right)$ indicates that there was an overall effect of practice on performance. In particular, RTs were faster in the second session $(501 \mathrm{~ms})$ than in the first session (531 ms). There were no higher-order interactions of the order of drug administration with any of the other factors in the analysis of variance, indicating that the practice effect (the reduction in $\mathrm{RT}$ in the second session) was not specific to any single attention condition.

To further characterize the effect of order, we measured the effect of donepezil on cueing separately in the two groups (subjects who were administered donepezil in the first session and those who were administered donepezil in the second session). In both groups, there was a consistent facilitatory effect of donepezil on the cueing effect in the voluntary attention condition (donepezil first: $11.8 \mathrm{~ms}$; donepezil second: $6.4 \mathrm{~ms}$ ), and the difference between the groups was not significant (two-tailed $t$-test, $t_{9}=0.43$, $p=0.68$ ). For involuntary attention, donepezil had a small effect on the cueing effect, and the drug effect was in opposite directions in the two groups (donepezil first: $2.6 \mathrm{~ms}$; donepezil second: $-4.8 \mathrm{~ms}$ ). The difference between 
the groups was again not statistically significant (two-tailed $t$-test, $t_{9}=0.74, p=0.48$ ). Finally, there were no significant differences between the groups in any of the eight experimental conditions (combinations of drug, SOA, and target location).

Subjects were instructed to respond as quickly and as accurately as they could. Performance was well above $90 \%$ correct in all conditions (mean: $94.5 \%$ ), and the analysis of RT was restricted to trials in which a correct response was made. Nevertheless, we measured the effects of voluntary and involuntary attention and cholinergic enhancement on behavioral accuracy. Similar to the RT findings, there was a significant interaction of target location and SOA on percent correct $\left(\mathrm{F}_{1,18}=27.4, p<0.01\right)$. This effect was modest in magnitude, resulting in a cueing effect of $1.5 \%$ correct in the long SOA blocks (greater accuracy for opposite location compared with cue location trials). In the short SOA blocks, the cueing effect on accuracy was $-1.7 \%$ correct, indicating a decrease in performance for targets at the opposite location relative to cue location, due to capture of involuntary attention by the cue. Importantly, administration of the drug had no overall effect on accuracy $\left(\mathrm{F}_{1,18}=1.3, p=0.3\right)$, and there was no significant interaction of drug administration with either target location or SOA in the analysis of performance accuracy.

\section{DISCUSSION}

We have found that pharmacological enhancement of the cholinergic system in healthy human subjects increases the effects of voluntary but not involuntary attention. These results provide further evidence that voluntary and involuntary attention have different neural substrates (Kincade et al, 2005; Landau et al, 2007; Esterman et al, 2008). Allocation of voluntary attention can improve processing at an attended location at the cost of impaired processing in other locations (Bashinski and Bacharach, 1980; Posner et al, 1980). Our results suggest that cholinergic enhancement specifically increases the benefits of voluntary attention for processing stimuli at the attended location. Another possible explanation of our findings is that donepezil causes a shift in baseline performance, accompanied by a change in both the costs and the benefits due to voluntary attention. As we did not include a neutral condition in which the cue provided no information about subsequent target location, we cannot determine whether there is a pharmacological effect on baseline performance. However, we found no effect of donepezil on overall RTs (across all conditions). In addition, donepezil reduced RTs only when targets appeared at the location at which voluntary attention was directed. We therefore favor the more parsimonious explanation: cholinergic enhancement causes a specific increase in the processing benefits due to voluntary attention.

A number of studies have assessed the effects of the ACh receptor agonist nicotine in spatial cueing tasks. Some have found a reduction in the size of the validity effect (RT difference between valid and invalid trials) in humans (Meinke et al, 2006; Vossel et al, 2008), whereas others have not (Griesar et al, 2002; Giessing et al, 2006). Consistent with our results, Meinke et al, 2006 reported an effect of nicotine on voluntary but not involuntary attention. However, unlike our finding that donepezil selectively increased the benefits of voluntary attention, nicotine decreased the benefits (valid trials), as well as the costs (invalid trials) of voluntary attention (Meinke et al, 2006). Moreover, nicotine reduced overall RT (Griesar et al, 2002; Meinke et al, 2006), suggesting a possible nonspecific effect of this drug, whereas there was no main effect of donepezil in the present study. It is difficult to directly compare the results of the two studies, because in the Meinke et al, 2006 study, voluntary and involuntary attention trials differed in the type of cue (central versus peripheral), SOA, and the proportion of valid and invalid trials. In contrast, our design used identical cues, targets, and cue validity, with involuntary and voluntary attention trials differing only in SOA and target duration.

In addition, nicotine is an agonist of only the nicotinic subtype of $\mathrm{ACh}$ receptors, and physiological evidence suggests that the effects of voluntary visual spatial attention on activity of neurons in primary visual cortex are mediated by muscarinic ACh receptors (Herrero et al, 2008). Receptor agonists and antagonists interact directly with subtypes of $\mathrm{ACh}$ receptors at all synapses where those receptor subtypes are located, independent of the amount of endogenous activity at those synapses. In contrast, cholinesterase inhibitors preferentially enhance cholinergic transmission at those synapses that are endogenously releasing ACh during performance of a given task. In addition, donepezil is relatively selective for the form of cholinesterase expressed in the central nervous system (Rogers et al, 1991; Kosasa et al, 1999). It is therefore more physiologically relevant than receptor agonists and antagonists for the study of the role of the cholinergic system in modulation of behavior and neural processing.

Cholinesterase inhibitors such as donepezil and physostigmine increase synaptic levels of ACh in the synaptic cleft and are frequently used in humans to mitigate cognitive decline in Alzheimer's disease. Some studies have found that cholinesterase inhibitors significantly affect measures of cognitive function and quality of life in patients with Alzheimer's disease (Mohs et al, 2001; Boada-Rovira et al, 2004), but the utility of their administration is still controversial (Courtney et al, 2004; Raschetti et al, 2007). Therefore, it would be beneficial to have a more complete understanding of the specific aspects of cognition and behavioral performance that are pharmacologically enhanced by increases in synaptic ACh.

Physostigmine administration has been reported to improve performance on a voluntary visual attention task (Bentley et al, 2004), but reduced RTs were observed in this attention task and other visual tasks. This generalized improvement suggests that physostigmine may have produced an increase in vigilance and/or arousal that was not specific to visual spatial attention. In the present study, no generalized effect was found, suggesting that the results are due to effects of donepezil on attention and not on vigilance and/or arousal. These differences between the effects of physostigmine and donepezil on overall RT may also stem from the relative selectivity of donepezil for the type of cholinesterase found in the central nervous system (Rogers et al, 1991). 
Although ACh has been proposed to increase signal-tonoise ratio (SNR) in cortical neurons (Sato et al, 1987), this idea remains controversial. One measure of SNR is the magnitude of a neuron's stimulus-driven response relative to its spontaneous baseline firing rate. This type of SNR has been observed to increase (Sillito and Kemp, 1983; Sato et al, 1987) or decrease (Zinke et al, 2006) after local ACh administration to visual cortex of anesthetized animals. In addition, ACh has been reported to enhance selectivity of visual cortical neurons for stimulus orientation and direction (Sillito and Kemp, 1983), whereas others observed a reduction in this type of stimulus selectivity in visual cortex (Sato et al, 1987; Zinke et al, 2006).

The increase in the benefits conferred by voluntary attention after cholinergic enhancement could be a result of at least two possible physiological mechanisms. The first mechanism is a direct 'bottom-up' modulation of visual processing of the target stimulus in early visual cortical areas. Animal studies have shown that ACh increases thalamocortical synaptic transmission relative to lateral intracortical connections (Giocomo and Hasselmo, 2007). In addition, $\mathrm{ACh}$ reduces the lateral spread of excitatory activity in rat visual cortical slices (Kimura et al, 1999) and decreases the optimal stimulus length for cells in marmoset area V1 (Roberts et al, 2005). In humans, administration of donepezil decreases the spatial spread of excitatory fMRI visual responses in early visual cortex, consistent with a reduction in excitatory receptive field size in visual cortical neurons (Silver et al, 2008). Thus, increasing ACh levels may result in a more reliable representation of the stimulus in visual cortex. We hypothesize that voluntary attention may cause spatially and/or temporally specific increases in ACh levels in cortical regions that contain neurons representing the attended visual field location. The resulting boost in thalamocortical transmission may act to gate sensory signals in these neurons, thereby facilitating processing of stimuli at the attended location. This hypothesis is consistent with a recent study that found that ACh can be released in cortex in a transient and spatially specific manner (Parikh et al, 2007).

A second possible physiological mechanism ('top-down') is an increased effect of $\mathrm{ACh}$ in attention control areas in frontal and/or parietal cortex. Local increases in ACh concentrations in prefrontal cortex correlate with behavioral performance in a task that requires attention (Parikh et al, 2007). Increased cholinergic neurotransmission in frontal cortex may potentiate activity in frontal and parietal cortical areas that have been associated with control of voluntary attention (Serences and Yantis, 2006) and may consequently improve performance in the voluntary attention condition. Further research is needed to distinguish these two possible mechanisms of cholinergic modulation of voluntary attention.

In conclusion, we have demonstrated that cholinergic enhancement with donepezil selectively augments voluntary attention with no measurable effects on involuntary attention. These findings suggest that voluntary and involuntary attentions are associated with different neural mechanisms. Finally, these results shed light on the role of the cholinergic system in modulation of cognitive functions in humans and demonstrate the potential to enhance these functions through pharmacological manipulations.

\section{ACKNOWLEDGEMENTS}

Jon Kelvey helped in the collection and analysis the data. Funding support was provided by the National Eye Institute (grant R21-EY17926 to MAS) and by the Hellman Family Faculty Fund at the University of California, Berkeley.

\section{DISCLOSURE}

The authors declare no conflict of interest.

\section{REFERENCES}

Arnold HM, Burk JA, Hodgson EM, Sarter M, Bruno JP (2002). Differential cortical acetylcholine release in rats performing a sustained attention task versus behavioral control tasks that do not explicitly tax attention. Neuroscience 114: 451-460.

Bashinski HS, Bacharach VR (1980). Enhancement of perceptual sensitivity as the result of selectively attending to spatial locations. Percept Psychophys 28: 241-248.

Bentley P, Husain M, Dolan RJ (2004). Effects of cholinergic enhancement on visual stimulation, spatial attention, and spatial working memory. Neuron 41: 969-982.

Boada-Rovira M, Brodaty H, Cras P, Baloyannis S, Emre M, Zhang $\mathrm{R}$ et al (2004). Efficacy and safety of donepezil in patients with Alzheimer's disease: results of a global, multinational, clinical experience study. Drugs Aging 21: 43-53.

Brainard DH (1997). The psychophysics toolbox. Spat Vis 10: 433-436.

Courtney C, Farrell D, Gray R, Hills R, Lynch L, Sellwood E et al (2004). Long-term donepezil treatment in 565 patients with Alzheimer's disease (AD2000): randomised double-blind trial. Lancet 363: 2105-2115.

Esterman M, Prinzmetal W, DeGutis J, Landau A, Hazeltine E, Verstynen T et al (2008). Voluntary and involuntary attention affect face discrimination differently. Neuropsychologia 46: 1032-1040.

Giessing C, Thiel CM, Rösler F, Fink GR (2006). The modulatory effects of nicotine on parietal cortex activity in a cued target detection task depend on cue reliability. Neuroscience 137: 853-864.

Giocomo LM, Hasselmo ME (2007). Neuromodulation by glutamate and acetylcholine can change circuit dynamics by regulating the relative influence of afferent input and excitatory feedback. Mol Neurobiol 36: 184-200.

Griesar WS, Zajdel DP, Oken BS (2002). Nicotine effects on alertness and spatial attention in non-smokers. Nicotine Tob Res 4: 185-194.

Herrero JL, Roberts MJ, Delicato LS, Gieselmann MA, Dayan P, Thiele A (2008). Acetylcholine contributes through muscarinic receptors to attentional modulation in V1. Nature 454: 1110-1114.

Keppel G, Wickens TD (2004). Design and Analysis: A Researcher's Handbook. 4th edn. Pearson Education: Upper Saddle River, NJ.

Kimura F, Fukuda M, Tsumoto T (1999). Acetylcholine suppresses the spread of excitation in the visual cortex revealed by optical recording: possible differential effect depending on the source of input. Eur J Neurosci 11: 3597-3609.

Kincade JM, Abrams RA, Astafiev SV, Shulman GL, Corbetta M (2005). An event-related functional magnetic resonance imaging study of voluntary and stimulus-driven orienting of attention. J Neurosci 25: 4593-4604.

Kosasa T, Kuriya Y, Matsui K, Yamanishi Y (1999). Inhibitory effects of donepezil hydrochloride (E2020) on cholinesterase activity in brain and peripheral tissues of young and aged rats. Eur J Pharmacol 386: 7-13.

Landau AN, Esterman M, Robertson LC, Bentin S, Prinzmetal W (2007). Different effects of voluntary and involuntary attention on EEG activity in the gamma band. J Neurosci 27: 11986-11990. 
Loftus GR, Masson MEJ (1994). Using confidence intervals in within-subject designs. Psychon Bull Rev 1: 476-490.

Meinke A, Thiel CM, Fink GR (2006). Effects of nicotine on visuospatial selective attention as indexed by event-related potentials. Neuroscience 141: 201-212.

Mohs RC, Doody RS, Morris JC, Ieni JR, Rogers SL, Perdomo CA et al (2001). A 1-year, placebo-controlled preservation of function survival study of donepezil in AD patients. Neurology 57: 481-488.

Muir JL, Everitt BJ, Robbins TW (1994). AMPA-induced excitotoxic lesions of the basal forebrain: a significant role for the cortical cholinergic system in attentional function. J Neurosci 14: 2313-2326.

Parikh V, Kozak R, Martinez V, Sarter M (2007). Prefrontal acetylcholine release controls cue detection on multiple timescales. Neuron 56: 141-154.

Pelli DG (1997). The VideoToolbox software for visual psychophsyics: transforming numbers into movies. Spat Vis 10: 437-442.

Posner MI, Cohen Y, Rafal RD (1982). Neural systems control of spatial orienting. Philos Trans R Soc Lond B Biol Sci 298: 187-198.

Posner MI, Snyder CRR, Davidson BJ (1980). Attention and the detection of signals. J Exp Psychol Gen 109: 160-174.

Prinzmetal W, Landau AN (2010). Dissecting Spatial Visual Attention. In: Coltheart V (ed) Tutorials in Visual Cognition. Psychology Press: Hove, UK.

Prinzmetal W, McCool C, Park S (2005). Attention: reaction time and accuracy reveal different mechanisms. J Exp Psychol Gen 134: 73-92.

Prinzmetal W, Zvinyatskovskiy A, Gutierrez P, Dilem L (2008). Voluntary and involuntary attention have different consequences: the effect of perceptual difficulty. Q J Exp Psychol 62: 352-369.

Raschetti R, Albanese E, Vanacore N, Maggini M (2007). Cholinesterase inhibitors in mild cognitive impairment: a systematic review of randomised trials. PLoS Med 4: e338.

Roberts MJ, Zinke W, Guo K, Robertson R, McDonald JS, Thiele A (2005). Acetylcholine dynamically controls spatial integration in marmoset primary visual cortex. J Neurophysiol 93: 2062-2072.
Rogers SL, Friedhoff LT (1998). Pharmacokinetic and pharmacodynamic profile of donepezil $\mathrm{HCl}$ following single oral doses. Br J Clin Pharmacol 46(Suppl 1): 1-6.

Rogers SL, Yamanishi Y, Yamatsu K (1991). E2020 - The pharmacology of a piperidine cholinesterase inhibitor. In: Becker R, Giacobini E (eds). Cholinergic Basis for Alzheimer Therapy. Birkhäuser: Boston, MA.

Sarter M, Hasselmo ME, Bruno JP, Givens B (2005). Unraveling the attentional functions of cortical cholinergic inputs: interactions between signal-driven and cognitive modulation of signal detection. Brain Res Rev 48: 98-111.

Sato H, Hata Y, Masui H, Tsumoto T (1987). A functional role of cholinergic innervation to neurons in the cat visual cortex. J Neurophysiol 58: 765-780.

Serences JT, Yantis S (2006). Selective visual attention and perceptual coherence. Trends Cogn Sci 10: 38-45.

Sereno AB, Holzman PS (1996). Spatial selective attention in schizophrenic, affective disorder, and normal subjects. Schizophr Res 20: 33-50.

Sillito AM, Kemp JA (1983). Cholinergic modulation of the functional organization of the cat visual cortex. Brain Res 289: 143-155.

Silver MA, Ress D, Heeger DJ (2007). Neural correlates of sustained spatial attention in human early visual cortex. J Neurophysiol 97: 229-237.

Silver MA, Shenhav A, D'Esposito M (2008). Cholinergic enhancement reduces spatial spread of visual responses in human early visual cortex. Neuron 60: 904-914.

Vossel S, Thiel CM, Fink GR (2008). Behavioral and neural effects of nicotine on visuospatial attentional reorienting in nonsmoking subjects. Neuropsychopharmacology 33: 731-738.

Warner CB, Juola JF, Koshino H (1990). Voluntary allocation versus automatic capture of visual attention. Percept Psychophys 48: 243-251.

Yantis S, Jonides J (1990). Abrupt visual onsets and selective attention: voluntary versus automatic allocation. J Exp Psychol Hum Percept Perform 16: 121-134.

Zinke W, Roberts MJ, Guo K, McDonald JS, Robertson R, Thiele A (2006). Cholinergic modulation of response properties and orientation tuning of neurons in primary visual cortex of anaesthetized Marmoset monkeys. Eur J Neurosci 24: 314-328. 